\title{
Microbial Biotechnology Based Modified Yeast
}

\section{Suleimanova AD*, Bulmakova DS and Sharipova MR \\ Institute of Fundamental Medicine and Biology, Kazan Federal University, Russia}

*Corresponding author: Aliya Suleimanova, Institute of Fundamental Medicine and Biology, Kazan Federal University, Kazan, Kremlyovskaya Street 18, Russia, 420008, Tel: (+927) 4342839; Email: aliya.kzn@gmail.com

\section{Mini Review \\ Volume 5 Issue 1}

Received Date: November 29, 2019

Published Date: January 03, 2020

DOI: $10.23880 /$ oajmb-16000155

\section{Abstract}

The production of heterologous proteins, lipids and chemicals based on modified yeast is a rapidly developing area of microbial biotechnology. Methods of metabolic engineering and new technologies for editing genomes are used to create a fundamentally new high-performance industrially significant yeast strains with new properties.

Keywords: Yeast; Genome-Editing Technologies; Metabolic Engineering

Abbreviations: DSB: Double-Stranded Breaks; ZFN: Zinc-Finger Nucleases; CRISPR: Clustered Regularly Interspaced Palindromic Repeats; TALEN: Transcription Activator-Like Effector Nucleases.

\section{Introduction}

Yeast is a convenient model eukaryotic organism for genetic modification: besides their whole genome being sequenced, they are easily cultivated in a bioreactor and recognized as safe organisms. About $20 \%$ of all pharmaceutical proteins are obtained using S. cerevisiae [1]. Non-traditional yeast is also actively used as producers [2].

For targeted gene expression in yeasts, genomic editing methods are used. The Cre-loxP system is considered one of the first editing systems, consisting of cre-recombinase (cyclic recombinase) and short DNA sequences-LoxP sites. Cre-recombinase interacts with LoxP sites and specifically cuts a DNA fragment between them [3]. An alternative strategy is the introduction of DNA double-stranded breaks (DSBs) using endonucleases. Due to the negative effect of DSBs on cells, the intracellular repair pathways are aimed at its restoration. A repair matrix can only be used as a donor for homologous recombination provided it shows homology to the gap site. Tools for targeted DSBs include, but not limited to dimeric meganucleases, zinc-finger nucleases (ZFNs), transcription activator-like effector nucleases (TALENs), and clustered regularly interspaced palindromic repeats (CRISPRs) [4]. These nucleases create site-specific DSBs in the DNA of a specific part of the genome, which are repaired during the recombination process and thus, making targeted mutations successful.

Progress in the development of DNA-editing methods and the improvement of sequencing technologies have contributed to the development of metabolic engineering, which modifies the initial metabolic pathways and their regulation. This in turn makes way for obtaining microorganisms with a high level of target compound production. For instance, more than 100 different proteins were acquired using the yeast Kluyveromyces lactis, which is known to metabolize low-cost carbon sources [5]. Using the CRISPR-Cas9 method, a synthetic pathway for the formation of muconic acid was created for them [6]. The Kluyveromyces marxianus yeast produces ethyl acetate, bioethanol, fuselols and their esters [2]. By disrupting native genes and inducing the expression of heterologous genes, the strain K. marxianus produced ethanol from xylulose at a rate of $2.49 \mathrm{~g} / \mathrm{l} / \mathrm{h}$ [7]. The yeast Yarrowia lipolytica is characterized by its ability to synthesize lipids [8]. Using genomic editing, the yield of triacylglycerides was augmented [9]. By knocking 


\section{Open Access Journal of Microbiology \& Biotechnology}

out the TRP1 gene with the CRISPR-Cas9 system, a new strain producing triacetic acid lactone was developed [10].

The presence of a strong inducible system of protein expression and the nature of their glycosylation facilitated the use of the methylotrophic yeasts Hansenula polymorpha and Pichia pastoris for heterologous expression. Inactivation of the CAT8 gene in $\mathrm{H}$. polymorpha led to an increase in ethanol yield to $12.5 \mathrm{~g} /$ L [11]. In H. polymorpha, the CRISPR-Cas9 method was likewise used to introduce the TAL, 4CL, and STS genes for the high yield synthesis of resveratrol of up to $98 \mathrm{mg} /$ L [12]. In P. pastoris, a biosynthesis pathway for the nootkatone synthesis was developed [13]. Genetic modification of amino acid biosynthesis pathway in $\mathrm{P}$. pastoris allowed the strain to synthesize up to $2.22 \mathrm{~g} / \mathrm{l}$ of isobutanol, which was 43 times higher than the level of its production in the non-mutant strain [14].

With the improvement of sequencing methods and genome-editing tools, it is possible to eliminate the disadvantages of wild-type strains to obtain the desired phenotypes and significantly increase the productivity of industrially significant strains.

\section{References}

1. Neilsen J (2013) Production of biopharmaceutical proteins by yeast: advances through metabolic engineering. Bioengineered 4(4): 207-211.

2. Lobs AK, Schwartz C, Wheeldon I (2017) Genome and metabolic engineering in non-conventional yeasts: current advances and applications. Synth Syst Biotechnol 2(3): 198-207.

3. Pan R, Zhang J, Shen W, Tao ZQ, Li SP, et al. (2011) Sequential deletion of Pichia pastoris genes by a selfexcisable cassette. Fems Yeast Res 11(3): 292-298.

4. Liu Z, Liang Y, Ang EL, Zhao H (2017) A new era of genome integration-simply cut and paste. ACS Synth Biol 6(4): 601-609.

5. Spohner SC, Schaum V, Quitmann H, Czermak (2016) Kluyveromyces lactis: an emerging tool in biotechnology. J Biotechnol 222: 104-116.
6. Horwitz AA, Walter JM, Schubert MG, Kung SH, Hawkins K, et al. (2015) Efficient multiplexed integration of synergistic alleles and metabolic pathways in yeasts via CRISPR-Cas. Cell Syst 1(1): 8896.

7. Zhang XH, Tee LY, Wang XG, Huang QS, Yang SH, et al. (2015) Off-target effects in CRISPR/Cas9-mediated genome engineering. Mol Ther Nucleic Acids 4: 264.

8. Hussain MS, Rodriguez GM, Gao DF, Spagnuolo M, Gambill L, et al. (2016) Recent advances in bioengineering of the oleaginous yeast Yarrowia lipolytica. Aims Bioeng 3(4): 493-514.

9. Qiao KJ, Wasylenko TM, Zhou K, XU P, Stephanopoulos G (2017) Lipid production in Yarrowia lipolytica is maximized by engineering cytosolic redox metabolism. Nat Biotechnol 35(2): 173-177.

10. Markham KA, Palmer CM, Chwatko M, Wagner JM, Murray C, et al. (2018) Rewiring Yarrowia lipolytica toward triacetic acid lactone for materials generation. Proc Natl Acad Sci 115(9): 2096-2101.

11. Ruchala J, Kurylenko 00, Soontorngun N, Dmytruk KV, Sibirny AA (2017) Transcriptional activator Cat8 is involved in regulation of xylose alcoholic fermentation in the thermotolerant yeast Ogataea (Hansenula) polymorpha. Microb Cell Fact 16(1): 36.

12. Wang L, Deng A, Zhang Y, Liu S, Liang Y, et al. (2018) Efficient CRISPR-Cas9 mediated multiplex genome editing in yeasts. Biotechnol Biofuels 11: 277.

13. Wriessnegger T, Augustin P, Engleder M, Leitner E, Muller M, et al. (2014) Production of the sesquiterpenoid (p)-nootkatone by metabolic engineering of Pichia pastoris. Metab Eng 24: 18-29.

14. Siripong W, Wolf P, Kusumoputri TP, Downes JJ, Kocharin K, et al. (2018) Metabolic engineering of Pichia pastoris for production of isobutanol and isobutyl acetate. Biotechnol Biofuels 11: 1. 